PROCEEDINGS OF THE

AMERICAN MATHEMATICAL SOCIETY

Volume 127, Number 7, Pages 1941-1949

S 0002-9939(99)04855-8

Article electronically published on March 8, 1999

\title{
ON THE STRUCTURE OF PERIODIC MODULES OVER TAME ALGEBRAS
}

\author{
ANDRZEJ SKOWROŃSKI
}

(Communicated by Ken Goodearl)

\begin{abstract}
We describe the structure of stable tubes in the Auslander-Reiten quivers of tame algebras formed by indecomposable modules which do not lie on infinite short cycles. In particular, we prove that all algebras whose module categories have no infinite short cycles are of linear growth.
\end{abstract}

\section{INTRODUCTION}

Throughout this paper $K$ will denote a fixed algebraically closed field. By an algebra we mean a finite dimensional $K$-algebra (associative, with an identity), which we moreover assume (without loss of generality) to be basic and connected. Then $A \simeq K Q_{A} / I$ where $Q_{A}$ is the Gabriel quiver of $A$ and $I$ is an admissible ideal in the path algebra $K Q_{A}$ of $Q_{A}$. Equivalently, we will consider $A$ as a $K$ category whose class of objects is the set of vertices of $Q_{A}$. For an algebra $A$, we denote by $\bmod A$ the category of finite dimensional (over $K$ ) right $A$-modules, by $\operatorname{rad}(\bmod A)$ the Jacobson radical of $\bmod A$ and by $\operatorname{rad}^{\infty}(\bmod A)$ the infinite radical of $\bmod A$. Recall that $\operatorname{rad}(\bmod A)$ is generated by non-isomorphisms between indecomposable objects in $\bmod A$ and $\operatorname{rad}^{\infty}(\bmod A)$ is the intersection of all finite powers $\operatorname{rad}^{i}(\bmod A), i \geq 1$, of $\operatorname{rad}(\bmod A)$. By an $A$-module we mean an object of $\bmod A$. For a vertex $i$ of $Q_{A}$, we denote by $S_{A}(i)$ the simple $A$-module given by $i$, and by $P_{A}(i)$ (respectively, $I_{A}(i)$ ) the projective cover (respectively, injective envelope) of $S_{A}(i)$ in $\bmod A$. Moreover, we denote by $D$ the standard duality $\operatorname{Hom}_{K}(-, K)$ on $\bmod A$.

We shall denote by $\Gamma_{A}$ the Auslander-Reiten quiver of $A$ and by $D$ Tr and $\operatorname{Tr} D$ the Auslander-Reiten translations in $\Gamma_{A}$. We do not distinguish between an indecomposable $A$-module and the vertex of $\Gamma_{A}$ corresponding to it. By a component of $\Gamma_{A}$ is meant a connected component of $\Gamma_{A}$. The support algebra $\operatorname{supp} \mathcal{C}$ of a component $\mathcal{C}$ of $\Gamma_{A}$ is the full subcategory of $A$ given by all objects $i$ such that $S_{A}(i)$ is a composition factor of a module in $\mathcal{C}$. The annihilator $\operatorname{ann}_{A} \mathcal{C}$ of a component $\mathcal{C}$ of $\Gamma_{A}$ is the intersection of the annihilators of all modules from $\mathcal{C}$. A component $\mathcal{C}$ of $\Gamma_{A}$ is said to be sincere (respectively, faithful) if $\operatorname{supp} \mathcal{C}=A$ (respectively, $\operatorname{ann}_{A} \mathcal{C}=0$ ). Clearly, any faithful component is sincere. A component in $\Gamma_{A}$ of the form $\mathbb{Z} A_{\infty} /\left(\tau^{r}\right), r \geq 1$, is said to be a stable tube (of rank $r$ ). Therefore, a stable tube of rank $r$ in $\Gamma_{A}$ is an infinite component consisting of

Received by the editors May 28, 1997 and, in revised form, September 26, 1997.

1991 Mathematics Subject Classification. Primary 16G60, 16G70.

The research was supported by the Polish Scientific Grant KBN No. 2P03A 02008. 
$D$ Tr-periodic indecomposable $A$-modules having period $r$. It is known [10] that any stable component of $\Gamma_{A}$ containing a $D$ Tr-periodic module is a stable tube.

A short cycle in $\bmod A$ is a sequence $X \stackrel{f}{\longrightarrow} Y \stackrel{g}{\longrightarrow} X$ of non-zero maps in $\operatorname{rad}(\bmod A)$ between indecomposable $A$-modules [20]. Such a short cycle is said to be finite if $f$ and $g$ do not belong to $\operatorname{rad}^{\infty}(\bmod A)$, and otherwise to be infinite. It was proved in [20], [9] that an indecomposable $A$-module $Y$ lies on a short cycle if and only if $Y$ is the middle of a short chain $Z \rightarrow Y \rightarrow D \operatorname{Tr} Z$ of non-zero maps and $Z$ indecomposable (the concept introduced in [2]). Indecomposable $A$-modules which are not on short cycles are uniquely determined by their composition factors [20]. Module categories without infinite short cycles have been investigated in [18], $[26],[27],[28],[30]$. We note also that the module categories without any short cycle have only finitely many pairwise non-isomorphic indecomposable modules [9].

From the Tame and Wild Theorem [7] the class of algebras may be divided into two disjoint classes. One class consists of wild algebras whose representation theory is as complicated as the study of finite dimensional vector spaces over $K$ together with two non-commuting endomorphisms, for which the classification is a well-known unsolved problem. The second class is formed by the tame algebras for which the indecomposable modules occur, in each dimension $d$, in a finite number of discrete and a finite number of one-parameter families. Hence, we can hope to classify modules only for tame algebras. More precisely, an algebra $A$ is said to be tame if, for any dimension $d$, there exists a finite number of $K[x]-A$-bimodules $M_{i}$, $1 \leq i \leq n_{d}$, which are finitely generated and free as left $K[x]$-modules, and all but a finite number of isomorphism classes of indecomposable $A$-modules of dimension $d$ are of the form $K[x] /(x-\lambda) \otimes_{K[x]} M_{i}$ for some $\lambda \in K$ and some $i$. Let $\mu_{A}(d)$ be the least number of $K[x]-A$-bimodules satisfying the above conditions for $d$. Then $A$ is said to be of linear growth (respectively, domestic) if there exists a positive integer $m$ such that $\mu_{A}(d) \leq m d$ (respectively, $\mu_{A}(d) \leq m$ ) for all $d \geq 1$ (see [24], $[6]$ ). From the validity of the second Brauer-Thrall conjecture we know that $A$ is representation-finite $(\bmod A$ has only finitely many isoclasses of indecomposable objects) if and only if $\mu_{A}(d)=0$ for any $d \geq 1$. Moreover, it was proved in [5] that if an algebra $A$ is tame then, for any dimension $d$, all but a finite number of isomorphism classes of indecomposable $A$-modules of dimension $d$ lie in stable tubes of rank 1. Hence, one of the crucial open problems in the representation theory of algebras is to describe the support algebras of stable tubes in the Auslander-Reiten quivers of tame algebras.

In the paper we are interested in the structure of tame algebras $A$ whose stable tubes behave well in the category $\bmod A$. In Section 1 we prove that if the Auslander-Reiten quiver of a tame algebra $A$ contains a sincere stable tube $\mathcal{T}$ consisting of modules which do not lie on infinite short cycles then $A$ is either tame concealed or tubular (in the sense [22]), and the tube $\mathcal{T}$ is faithful. Recall that if $B$ is a tame concealed algebra then, by $[22,(4.3)]$,

$$
\Gamma_{B}=\mathcal{P}_{0} \vee \mathcal{T}_{0} \vee \mathcal{Q}_{0}
$$

where $\mathcal{P}_{0}$ is a preprojective component, $\mathcal{Q}_{0}$ is a preinjective component, and $\mathcal{T}_{0}$ is a $\mathbb{P}_{1}(K)$-family of faithful stable tubes. If $B$ is tubular, then, by $[22,(5.2)]$,

$$
\Gamma_{B}=\mathcal{P}_{0} \vee \mathcal{T}_{0} \vee\left(\bigvee_{q \in \mathbb{Q}^{+}} \mathcal{T}_{q}\right) \vee \mathcal{T}_{\infty} \vee \mathcal{Q}_{\infty}
$$


where $\mathcal{P}_{0}$ is a preprojective component, $\mathcal{T}_{0}$ is a $\mathbb{P}_{1}(K)$-family of ray tubes, $\mathcal{T}_{\infty}$ is a $\mathbb{P}_{1}(K)$-family of coray tubes, $\mathcal{Q}_{\infty}$ is a preinjective component, and, for each $q \in \mathbb{Q}^{+}$, $\mathcal{T}_{q}$ is a $\mathbb{P}_{1}(K)$-family of faithful stable tubes. Moreover, the module categories of tame concealed algebras and tubular algebras do not contain infinite short cycles. In Section 2 we prove that the above families of faithful stable tubes exhaust the stable tubes of arbitrary module categories without infinite short cycles. In particular, we get that an algebra $A$ for which every short cycle in $\bmod A$ is finite has linear growth, and it is domestic if and only if all but finitely many components of $\Gamma_{A}$ are stable tubes of rank 1 , or equivalently $\operatorname{rad}^{\infty}(\bmod A)$ is nilpotent.

For basic background on the representation theory applied here we refer to [3] and $[22]$.

\section{TAme Algebras With Sincere Stable TUBES}

Let $A$ be an algebra. For an $A$-module $M$, we denote by $\operatorname{pd}_{A} M$ (respectively, $\operatorname{id}_{A} M$ ) the projective dimension (respectively, injective dimension) of $M$. Moreover, we denote by gl. $\operatorname{dim} A$ the global dimension of $A$. Then $A$ is said to be quasi-tilted if gl. $\operatorname{dim} A \leq 2$ and for any indecomposable $A$-module $X$ we have $\operatorname{pd}_{A} X \leq 1$ or $\operatorname{id}_{A} X \leq 1$. It is shown in [11] that an algebra $A$ is quasi-tilted if and only if $A$ is the endomorphism $\operatorname{ring} \operatorname{End}_{\mathcal{H}}(T)$ of a tilting object in a hereditary abelian $K$-category $\mathcal{H}$. We refer to [11] for basic properties of quasi-tilted algebras. Recently, the author described in [31] completely the structure of all tame quasi-tilted algebras. The structure of wild quasi-tilted algebras is not known. It is expected that any quasi-tilted algebra is tilted or of canonical type [15]. Finally, we mention that the Auslander-Reiten quiver of any quasi-tilted algebra of canonical type admits a tubular family consisting of modules which do not lie on infinite short cycles. Moreover, the Auslander-Reiten quiver of a quasi-tilted algebra of canonical type contains a faithful (equivalently, sincere) stable tube if and only if $A$ is concealed-canonical (see [15]).

Proposition 1.1. Let $A$ be an algebra whose Auslander-Reiten quiver contains a faithful stable tube $\mathcal{T}$ consisting of modules which do not lie on infinite short cycles. Then $A$ is quasi-tilted.

Proof. It is well-known that a stable tube in $\Gamma_{A}$ is faithful if and only if it contains a faithful indecomposable module. Let $M$ be a faithful indecomposable module in $\mathcal{T}$. Then there are positive integers $r$ and $s$, a monomorphism $A_{A} \rightarrow M^{r}$, and an epimorphism $M^{s} \rightarrow D(A)_{A}$. Then it follows from [25, Corollary 5.8] that gl. $\operatorname{dim} A \leq 2$, and, by [25, Lemma 5.9], that $\operatorname{pd}_{A} Z \leq 1$ and $\operatorname{id}_{A} Z \leq 1$ for any indecomposable module $Z$ in $\mathcal{T}$. Let $X$ be an indecomposable $A$-module with $\operatorname{id}_{A} X=2$. We shall prove that the $\operatorname{pd}_{A} X \leq 1$. By the above remark, $X$ does not belong to $\mathcal{T}$. Consider an Auslander-Reiten sequence

$$
0 \rightarrow X \rightarrow E \rightarrow \operatorname{Tr} D X \rightarrow 0
$$

in $\bmod A$. Since $\operatorname{id}_{A} X \geq 2$, we have $\operatorname{Hom}_{A}\left(\operatorname{Tr} D X, A_{A}\right) \neq 0$ (see [22, (2.4)]) and hence $\operatorname{Hom}_{A}(\operatorname{Tr} D X, M) \neq 0$, because there is a monomorphism $A_{A} \rightarrow M^{r}$. We claim that $\operatorname{pd}_{A} \operatorname{Tr} D X \leq 1$. Indeed, if it is not the case, then $\operatorname{Hom}_{A}\left(D(A)_{A}, X\right) \neq$ 0 , and so $\operatorname{Hom}_{A}(M, X) \neq 0$, because there is an epimorphism $M^{s} \rightarrow D(A)_{A}$. Hence, we get a short chain $\operatorname{Tr} D X \rightarrow M \rightarrow X$ and, by [20, Theorem 1.6], a short cycle $N \rightarrow M \rightarrow N$, where $N$ is either an indecomposable direct summand of $E$ or $N=\operatorname{Tr} D X$. Observe that this short cycle is infinite, because $X$ does 
not belong to $\mathcal{T}$ and $M$ is from $\mathcal{T}$. This contradicts our assumption on $\mathcal{T}$, and hence $\operatorname{pd}_{A} \operatorname{Tr} D X \leq 1$. Since gl. $\operatorname{dim} A \leq 2$, the functor $\operatorname{Ext}_{A}^{2}(-, A / \operatorname{rad} A)$ is right exact. Let $Y$ be an indecomposable direct summand of $E$. If an irreducible map

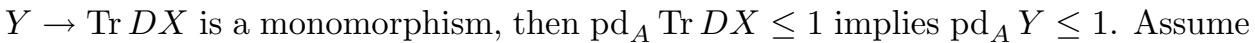
we have an irreducible epimorphism $Y \rightarrow \operatorname{Tr} D X$. Then $\operatorname{Hom}_{A}(\operatorname{Tr} D X, M) \neq 0$ implies $\operatorname{Hom}_{A}(Y, M) \neq 0$. We claim that $\operatorname{pd}_{A} Y \leq 1$. Indeed, if $\operatorname{pd}_{A} Y \geq 2$ then $\operatorname{Hom}_{A}\left(D(A)_{A}, D \operatorname{Tr} Y\right) \neq 0$, and consequently $\operatorname{Hom}_{A}(M, D \operatorname{Tr} Y) \neq 0$, because there is an epimorphism $M^{s} \rightarrow D(A)_{A}$. Hence we get a short chain $Y \rightarrow M \rightarrow$ $D \operatorname{Tr} Y$, and, applying again [20, Theorem 1.6], a short cycle $U \rightarrow M \rightarrow U$ where either $U=Y$ or $U$ is an indecomposable direct summand of the middle term of an Auslander-Reiten sequence

$$
0 \rightarrow D \operatorname{Tr} Y \rightarrow F \rightarrow Y \rightarrow 0
$$

Since $U$ does not lie in $\mathcal{T}$, we have an infinite short cycle $U \rightarrow M \rightarrow U$ with $M$ lying in $\mathcal{T}$, a contradiction. Therefore, we proved that $\operatorname{pd}_{A} Y \leq 1$, and hence $\operatorname{pd}_{A} E \leq 1$. Since $\operatorname{Ext}_{A}^{2}(-, A / \operatorname{rad} A)$ is right exact and we have a monomorphism $X \rightarrow E$, we get $\operatorname{pd}_{A} X \leq 1$. This proves that $A$ is quasi-tilted.

We shall prove now the following characterization of tame concealed and tubular algebras.

Theorem 1.2. Let $A$ be an algebra. The following conditions are equivalent:

(i) A is tame and $\Gamma_{A}$ contains a sincere stable tube consisting of modules which do not lie on infinite short cycles.

(ii) A is either tame concealed or tubular.

Proof. The implication (ii) $\Rightarrow$ (i) follows from $[22,(4.3)$ and (5.2)]. We shall prove that (i) implies (ii). The proof will be done in several steps.

(1) Let $A=K Q_{A} / I$, and assume that $\Gamma_{A}$ contains a sincere stable tube $\mathcal{T}$ consisting of modules which do not lie on infinite short cycles. Denote by $J$ the annihilator of $\mathcal{T}$ in $A$. Consider the factor algebra $B=A / J$. Clearly, $B$ is tame and $\mathcal{T}$ is a faithful stable tube of $\Gamma_{B}$ consisting of modules which do not lie on infinite short cycles. From Proposition 1.1 we conclude that $B$ is quasi-tilted. It is shown in [31] that a quasi-tilted algebra $\Lambda$ is tame if and only if $\Lambda$ is tame tilted or a tame semi-regular branch enlargement of a tame concealed algebra. Invoking now the known structure of module categories of tame tilted algebras [13], [22] and tame semi-regular branch enlargements of tame concealed algebras [15], [31], and the fact that $\mathcal{T}$ is a faithful stable tube of $\Gamma_{B}$, we conclude that $B$ is either tame concealed or tubular. In particular, $B$ is a tame tubular extension of a tame concealed algebra $C$.

(2) We shall prove now that $Q_{A}=Q_{B}$. Since $\mathcal{T}$ is a sincere tube of both $\Gamma_{A}$ and $\Gamma_{B}$, we know that $Q_{A}$ and $Q_{B}$ have the same sets of vertices. Suppose $Q_{A}$ contains an arrow $i \stackrel{\alpha}{\longrightarrow} j$ which is not an arrow of $Q_{B}$. Denote by $\Lambda$ the algebra $K Q_{A} / L$ where $L$ is the ideal of $K Q_{A}$ generated by $I$, all arrows of $Q_{A} \backslash Q_{B}$ except $\alpha$, and all path in $Q_{A}$ of the forms $\alpha \gamma$ and $\delta \alpha$. Clearly, $\Lambda$ is a factor algebra of $A$ and $B$ is a factor algebra of $\Lambda$. In particular, $\mathcal{T}$ is a stable tube of $\Gamma_{\Lambda}$ consisting of modules which do not lie on infinite short cycles. Let $E$ be the $B$-B-bimodule $D\left(S_{B}(i)\right) \otimes_{K} S_{B}(j)$. Consider the following locally finite dimensional $K$-algebra 
(without identity)

$$
\widetilde{\Lambda}=\left[\begin{array}{ccccc}
\ddots & \ddots & & & \\
& B_{m+1} & E_{m} & & \\
& & B_{m} & E_{m-1} & \\
& & & B_{m-1} & \ddots \\
& & & & \ddots
\end{array}\right]
$$

with $B_{m}=B$ on the main diagonal, $E_{m}=E$, for all $m \in \mathbb{Z}$, and zeros elsewhere. The elements of $\widetilde{\Lambda}$ are all matrices with only finitely many non-zero entries, and the multiplication is given by the $B-B$-bimodule structure of $E$ and the zero map $E \otimes_{B} E \rightarrow 0$. The identity maps $B_{m} \rightarrow B_{m+1}$ and $E_{m} \rightarrow E_{m+1}$ induce an automorphism $g$ of $\widetilde{\Lambda}$, and denote by $G$ the infinite cyclic group generated by $g$. Then we have a canonical Galois covering $F: \widetilde{\Lambda} \rightarrow \widetilde{\Lambda} / G=\Lambda$ with group $G$ (see [8]), because $\Lambda$ can be an identified with the algebra

$$
\left[\begin{array}{lll}
B & & E \\
& \Downarrow & \\
0 & & B
\end{array}\right]=\left\{\left[\begin{array}{ll}
b & e \\
0 & b
\end{array}\right] ; b \in B, e \in E=K\right\} .
$$

Denote by $F_{\lambda}: \bmod \widetilde{\Lambda} \rightarrow \bmod \Lambda$ the associated push-down functor. Since $G$ is torsion-free, $F_{\lambda}$ preserves the indecomposable modules $[8,(3.5)]$. We know that $B$ is either tame concealed or tubular. Hence, $\Gamma_{B}=\mathcal{P} \vee \mathcal{C} \vee \mathcal{Q}$, where $\mathcal{C}$ is a $\mathbb{P}_{1}(K)$-family of stable tubes, containing the tube $\mathcal{T}$, and separating $\mathcal{P}$ from $\mathcal{Q}$ (see $[22,(4.3)$ and $(5.2)]$ ). In particular, there are an indecomposable $B$-module $X$ in $\mathcal{P}$ with $\operatorname{Hom}_{B}\left(X, S_{B}(i)\right) \neq 0$ and an indecomposable $B$-module $Y$ in $\mathcal{Q}$ with $\operatorname{Hom}_{B}\left(S_{B}(j), Y\right) \neq 0$. Let $f: X \rightarrow S_{B}(i)$ and $h: S_{B}(j) \rightarrow Y$ be non-zero maps in $\bmod B$. Consider now the module $Z$ over the factor algebra

$$
\left[\begin{array}{cc}
B_{1} & E_{0} \\
0 & B_{0}
\end{array}\right]
$$

of $\widetilde{\Lambda}$ given by the triple $\left(X_{B_{1}}, Y_{B_{0}}, \varphi\right)$, where the homomorphism $\varphi: X \otimes_{B_{1}} E \rightarrow Y$ of right $B_{0}$-modules is the composition

$$
X \otimes_{B} E \stackrel{f \otimes 1}{\longrightarrow} S_{B}(i) \otimes_{B} E \stackrel{\sim}{\longrightarrow} S_{B}(j) \stackrel{h}{\longrightarrow} Y .
$$

Clearly, $Z$ is an indecomposable $\widetilde{\Lambda}$-module, and so we get an indecomposable $\Lambda$ module $N=F_{\lambda} Z$ having $X$ as a factor module and $Y$ as a submodule. Since $X$ belongs to $\mathcal{P}, \mathcal{Q}$ contains all indecomposable injective $B$-modules, and $\mathcal{C}$ separates $\mathcal{P}$ from $\mathcal{Q}$, we deduce that $\operatorname{rad}_{B}^{\infty}(X, U)=\operatorname{Hom}_{B}(X, U) \neq 0$ for some $U \in \mathcal{T}$. Dually, since $\mathcal{P}$ contains all indecomposable projective $B$-modules, $Y$ belongs to $\mathcal{Q}$, there exists $V \in \mathcal{T}$ such that $\operatorname{rad}_{B}^{\infty}(V, Y)=\operatorname{Hom}_{B}(V, Y) \neq 0$. Therefore, we have $\operatorname{rad}_{\Lambda}^{\infty}(N, U) \neq 0$ and $\operatorname{rad}_{\Lambda}^{\infty}(V, N) \neq 0$ for some $U, V \in \mathcal{T}$. But then there exists an infinite short cycle $N \rightarrow M \rightarrow N$ with $M$ from $\mathcal{T}$ (see [26, (3.3)]), which contradicts our assumption on $\mathcal{T}$. Hence, $Q_{A}=Q_{B}$.

(3) Finally, we shall show that $A=B$, or equivalently $J=0$. We have two cases to consider. Assume first that $B$ is tame concealed. Since $Q_{A}=Q_{B}$ and $A$ is tame, a simple inspection of the frames of tame concealed algebras presented in [4] or [12] shows that in fact $J=0$. Assume now that $B$ is tubular. Then $B$ is a tubular extension of a tame concealed algebra $C$ of tubular type $(2,2,2,2)$, 
$(3,3,3),(2,4,4)$, or $(2,3,6)$, and $Q_{C}$ is a convex subquiver of $Q_{B}=Q_{A}$. There are 10 infinite families of tubular algebras of tubular type $(2,2,2,2)$ whose bound quivers are presented (for example) in $[23,(3.3)]$. If $B$ is one of such algebras, then a simple checking shows that $J=0$ also in this case. Hence, we may assume that $B$ is of tubular type different from $(2,2,2,2)$. Suppose $J \neq 0$. Recall that $B$ is obtained from an iterated one-point extension $C\left[E_{1}\right]\left[E_{2}\right] \ldots\left[E_{t}\right]$ of $C$ by pairwise non-isomorphic simple regular $C$-modules $E_{1}, E_{2}, \ldots, E_{t}$, say with the extension vertices $\omega_{1}, \omega_{2}, \ldots, \omega_{t}$, by rooting some branches $K_{1}, K_{2}, \ldots, K_{t}$ at $\omega_{1}, \omega_{2}, \ldots, \omega_{t}$, respectively $[22,(4.7)]$. Moreover, the branches $K_{1}, K_{2}, \ldots, K_{t}$ are bound only by zero-relations of length $2[22,(4.4)]$. Since $A$ is tame, we deduce as above that $C$ is a (convex) subcategory of $A$. Since $Q_{A}=Q_{B}$, the full subcategory of $A$ given by the objects of $C$ and $\omega_{1}, \omega_{2}, \ldots, \omega_{t}$ is an iterated one-point extension $C\left[F_{1}\right]\left[F_{2}\right] \ldots\left[F_{t}\right]$, where $F_{1}, F_{2}, \ldots, F_{t}$ are $C$-modules having $E_{1}, E_{2}, \ldots, E_{t}$ as factor modules, respectively. For each $1 \leq i \leq t$, denote also by $L_{i}$ the full subcategory of $A$ given by the vertices of the branch $K_{i}$. It follows from our assumption $J \neq 0$ that $E_{i} \neq F_{i}$ or $L_{i} \neq K_{i}$ for some $1 \leq i \leq t$. Consider first the case when $E_{i} \neq F_{i}$ for some $i$. Denote by $D$ the full subcategory of $B$ given by all objects of $B$ except the objects of $K_{i}$. Since $B$ is of tubular type $(3,3,3),(2,4,4)$ or $(2,3,6), D$ is the corresponding domestic tubular extension of $C$, and $\Gamma_{D}$ contains pairwise different tubes $\mathcal{T}_{1}, \mathcal{T}_{2}, \mathcal{T}_{3}$ such that $F_{i}$ belongs to $\mathcal{T}_{1}, \mathcal{T}_{2}$ has at least 2 rays and $\mathcal{T}_{3}$ has at least 3 rays. Clearly, the one-point extension $R=D\left[F_{i}\right]$ is a factor algebra of $A$, and so $R$ is tame. Then $F_{i}$ does not contain direct summands from the preprojective component of $\Gamma_{C}$ (see $[21,(2.5)]$ ). Since the tubes in $\Gamma_{C}$ are pairwise orthogonal and $E_{i}$ is a factor module of $F_{i}$, we conclude that $F_{i}$ is a direct sum of modules from $\mathcal{T}_{1}$ and at least one indecomposable direct summand of $F_{i}$ has two direct successors in $\mathcal{T}_{1}$. Let $H$ be a tame hereditary algebra and $T$ a tilting $H$-module without preinjective direct summands such that $D=\operatorname{End}_{H}(T)$. Then the tubes $\mathcal{T}_{1}, \mathcal{T}_{2}, \mathcal{T}_{3}$ are the images by $\operatorname{Hom}_{H}(T,-)$ of the torsion parts, with respect to the torsion theory in $\bmod H$ given by $T$, of the corresponding stable tubes $\mathcal{T}_{1}^{\prime}, \mathcal{T}_{2}^{\prime}, \mathcal{T}_{3}^{\prime}$ of $\Gamma_{H}$. In particular, $F_{i}=\operatorname{Hom}_{H}\left(T, F_{i}^{\prime}\right)$ for an $H$-module $F_{i}^{\prime}$ being a direct sum of modules from $\mathcal{T}_{1}^{\prime}$, and one of the indecomposable direct summands of $F_{i}^{\prime}$ is not simple regular. Moreover, $H$ has the same tubular type as $D$. Invoking now Theorem 3 from $[21,(3.5)]$, we conclude that $H\left[F_{i}^{\prime}\right]$ is wild. But then $B\left[F_{i}\right]$ is also wild (see $[19,(3.2)]$ ), a contradiction. This shows that $E_{i}=F_{i}$. Suppose now that $L_{i} \neq K_{i}$. Then, since $Q_{A}=Q_{B}$, we conclude that $A$ has a factor algebra $A^{\prime}$ which is obtained from the one-point extension $D\left[F_{i}\right]$, with the extension vertex $\omega_{i}$, by rooting a hereditary quiver of the form

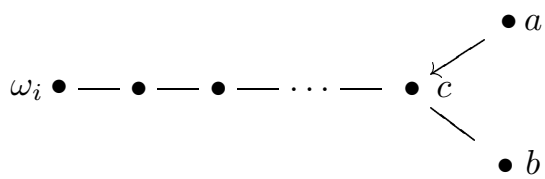

at $\omega_{i}$, where possibly $c=\omega_{i}$ and $\bullet \longrightarrow \bullet$ means $\bullet \longrightarrow \bullet$ or $\bullet \longleftarrow \bullet$. Denote by $A^{\prime \prime}$ the full subcategory of $A^{\prime}$ given by all objects except $a$. Then $A^{\prime}=A^{\prime \prime}\left[P_{A^{\prime \prime}}(c)\right]$, $A^{\prime \prime}$ is a domestic tubular extension of $C$, and $\Gamma_{A^{\prime \prime}}$ contains pairwise different tubes $\mathcal{T}_{1}^{*}, \mathcal{T}_{2}^{*}=\mathcal{T}_{2}, \mathcal{T}_{3}^{*}=\mathcal{T}_{3}$ such that $\mathcal{T}_{1}^{*}$ is obtained from $\mathcal{T}_{1}$ by ray insertions and $P_{A^{\prime \prime}}(c)$ is an indecomposable module in $\mathcal{T}_{1}^{*}$ having two direct successors. Then, as 
above, we conclude that $A^{\prime}=A^{\prime \prime}\left[P_{A^{\prime \prime}}(c)\right]$ is wild, and so $A$ is wild, a contradiction. Therefore, $A=B$, and so $A$ is either tame concealed or tubular.

Corollary 1.3. Let $A$ be a tame algebra and $\mathcal{T}$ a stable tube of $\Gamma_{A}$ consisting of modules which do not lie on infinite short cycles. Then there is an idempotent $e$ of $A$ such that $B=A / A e A$ is tame concealed or tubular, and $\mathcal{T}$ is a faithful stable tube of $\Gamma_{B}$.

Proof. There are pairwise orthogonal idempotents $e$ and $f$ of $A$ such that $A=e A \oplus$ $f A$ and the simple summands of $f A / f(\operatorname{rad} A)$ are exactly the simple composition factors of modules in $\mathcal{T}$. Put $B=A / A e A$. Then $\mathcal{T}$ is a sincere stable tube of $\Gamma_{B}$, and clearly consists entirely of $B$-modules which do not lie on infinite short cycles in $\bmod B$. From Theorem 1.2 we conclude that $B$ is either tame concealed or tubular. Moreover, $\mathcal{T}$ is a faithful stable tube of $\Gamma_{B}$, because by $[22,(4.3),(5.2)]$ it cogenerates $A_{A}$ and generates $D(A)_{A}$.

Remark 1.4. We note that the condition (i) in Theorem 1.2 cannot be deepened to: $A$ is tame and $\Gamma_{A}$ contains a sincere stable tube consisting of modules $X$ with $\operatorname{rad}^{\infty}(X, X)=0$, or equivalently a sincere generalized standard stable tube (see [26, (3.1)] or $[25,(5.3)])$. Indeed, let $H$ be a tame hereditary algebra and $A$ the trivial extension $H \ltimes D(H)$ of $H$ by the minimal injective cogenerator $D(H)$. Then $A$ is a symmetric tame (domestic) algebra, all tubes from the $\mathbb{P}_{1}(K)$-family of stable tubes in $\Gamma_{H}$ are generalized standard stable tubes of $\Gamma_{A}$, and consist of modules $X$ lying on infinite short cycles $P \rightarrow X \rightarrow P$ with $P$ indecomposable projective $A$-modules (see [1]).

\section{Module CATEgories Without infinite Short CyCles}

Let $A$ be an algebra such that all short cycles in $\bmod A$ are finite. Then $\operatorname{rad}^{\infty}(X, X)=0$ for any indecomposable $A$-module $X$, and so $A$ is tame (see [29, $(2.8)])$. It is shown in $[27,(4.3)]$ that $A$ is representation-infinite if and only if there is an idempotent $e$ of $A$ such that $A / A e A$ is tame concealed. Moreover, S. Liu has proved in $[18,(3.4)]$ that all but finitely many $D$ Tr-orbits in $\Gamma_{A}$ are periodic, and hence all but finitely many components of $\Gamma_{A}$ are stable tubes. The following theorem describes completely indecomposable modules in the stable tubes of $\Gamma_{A}$ and the representation type of $A$.

Theorem 2.1. Let $A$ be an algebra such that every short cycle in $\bmod A$ is finite. Then

(i) For any stable tube $\mathcal{T}$ of $\Gamma_{A}$, the support algebra $B$ of $\mathcal{T}$ is a factor algebra $A / A e A$ of $A$, for some idempotent $e$ of $A$, and is either tame concealed or tubular.

(ii) $A$ is of linear growth.

Proof. It follows from our assumption that $A$ is tame. Then (i) is a direct consequence of Corollary 1.3. For (ii), we may assume that $A$ is representation-infinite. Then, by [5, Corollary 4], for any dimension $d$, all but a finite number of isomorphism classes of indecomposable $A$-modules of dimension $d$ lie in stable tubes of rank 1. For module categories without infinite short cycles this fact is also proved in [26, Theorem 5.1]. We know from (i) that the support algebra of any stable tube of rank 1 in $\Gamma_{A}$ is a full subcategory of $A$ which is tame concealed or tubular, and 
hence of linear growth (see $[22,(4.3),(5.2)]$ and $[24,(3.6)]$ ). Since $A$ admits only finitely many full subcategories we infer that $A$ is of linear growth.

2.2. We note that the class of algebras $A$ for which all short cycles in $\bmod A$ are finite is large. The author has proved in $[30,(4.1)]$ that the module categories of all polynomial growth strongly simply connected algebras have no infinite short cycles. We mention that in such a case the Auslander-Reiten components different from stable tubes can be complicated (coils, multicoils). Moreover, the module categories of all tame quasi-tilted algebras do not contain infinite short cycles [31]. There are also algebras of infinite global dimension whose module categories do not contain infinite short cycles. Indeed, let $B$ be an arbitrary tilted algebra of Euclidean type, $\widehat{B}$ the repetitive algebra of $B$ and $\nu_{\widehat{B}}$ the Nakayama automorphism of $\widehat{B}$. Then, for $m \geq 3$, the algebra $A^{(m)}=\widehat{B} /\left(\nu_{\widehat{B}}^{m}\right)$ is selfinjective, domestic and every short cycle in $\bmod A^{(m)}$ is finite (see [23, Section 3]). Similarly, if $B$ is an arbitrary tubular algebra and $m \geq 3$, then $A^{(m)}=\widehat{B} /\left(\nu_{\widehat{B}}^{m}\right)$ is selfinjective, non-domestic of linear growth, and every short cycle in $\bmod A^{(m)}$ is finite.

Theorem 2.3. Let $A$ be an algebra such that every short cycle in $\bmod A$ is finite. The following conditions are equivalent:

(i) A is domestic.

(ii) A does not contain a tubular algebra as a full subcategory.

(iii) For any idempotent $e$ of $A, A / A e A$ is not a tubular algebra.

(iv) $\operatorname{rad}^{\infty}(\bmod A)$ is nilpotent.

(v) All but finitely many components of $\Gamma_{A}$ are stable tubes of rank 1.

Proof. It is known that every tubular algebra is non-domestic (see $[24,(3.6)]$ ) and hence (i) implies (ii) and (iii). Conversely, if (ii) or (iii) holds then it follows from the proof of Theorem 2.1 that $A$ is domestic. Moreover, if $C$ is a tame concealed algebra then all but finitely many components of $\Gamma_{C}$ are stable tubes of rank 1 $[22,(4.3)]$. Hence, the implication (ii) $\Rightarrow(\mathrm{v})$ is a direct consequence of Theorem 2.1 and the fact that all but finitely many components of $\Gamma_{A}$ are stable tubes [18, (3.4)]. Further, it was shown in $[14,(1.5)]$ that, if $B$ is a tubular algebra, then $\operatorname{rad}^{\infty}(\bmod B)$ is not nilpotent, and so (iv) implies (iii). Therefore, it remains to prove that (v) implies (iv). This follows from the proof of implication (iv) $\Rightarrow$ (iii) of Theorem 5.1 in [28] by invoking Theorem 2.1 and the following consequence of [16], [17]. Let $\mathcal{C}$ be a component of $\Gamma_{A}$ whose left stable part $\mathcal{C}_{l}$ contains a component $\mathcal{D}$ without oriented cycles. Then $\mathcal{D}$ admits a full translation subquiver $\mathcal{D}^{\prime}$ of the form $(-\mathbb{N}) \Delta$ which is closed under predecessors in $\mathcal{C}$ [17]. Since every short cycle in $\bmod A$ is finite and $\mathcal{D}^{\prime}$ has no oriented cycles, we conclude that $\mathcal{D}^{\prime}$ consists of modules which do not lie on short cycles. Hence, by $[16,(2.2)$ and $[2.3)], \mathcal{D}^{\prime}$ is a generalized standard full translation subquiver of $\Gamma_{A}$, that is, $\operatorname{rad}^{\infty}(X, Y)=0$ for any modules $X$ and $Y$ from $\mathcal{D}^{\prime}$.

\section{REFERENCES}

[1] I. Assem, J. Nehring and A. Skowroński, Domestic trivial extensions of simply connected algebras, Tsukuba J. Math. 13 (1989), 31-72. MR 90j:16043

[2] M. Auslander and I. Reiten, Modules determined by their composition factors, Illinois J. Math. 29 (1985), 280-301. MR 86i: 16032

[3] M. Auslander, I. Reiten and S. O. Smal $\varnothing$, Representation theory of Artin algebras, Cambridge studies in advanced math. 36, (Cambridge University Press, 1995). MR 96c:16015 
[4] K. Bongartz, Critical simply connected algebras, Manuscr. Math. 46 (1984), 117-136. MR 85j: 16026

[5] W. Crawley-Boevey, On tame algebras and bocses, Proc. London Math. Soc. 56 (1988), 451-483. MR 89c: 16028

[6] W. Crawley-Boevey, Tame algebras and generic modules, Proc. London Math. Soc. 63 (1991), 241-264. MR 92m:16019

[7] Y. A. Drozd, Tame and wild matrix problems, in: Representation Theory II, Lecture Notes in Math. 832 (Springer-Verlag, 1980), 242-258. MR 83b:16024

[8] P. Gabriel, The universal cover of a representation-finite algebra, in: Representations of Algebras, Lecture Notes in Math. 903 (Springer-Verlag, 1981), 68-105. MR 83f:16036

[9] D. Happel and S. Liu, Module categories without short cycles are of finite type, Proc. Amer. Math. Soc. 120 (1994), 371-375. MR 94d:16010

[10] D. Happel, U. Preiser and C. M. Ringel, Vingberg's characterization of Dynkin diagrams using subadditive functions with application to D Tr-periodic modules, in: Representation Theory II, Lecture Notes in Math. 832 (Springer-Verlag, 1980), 280-294. MR 82g:16027

[11] D. Happel, I. Reiten and S. O. Smalø, Tilting in abelian categories and quasi-tilted algebras, Memoirs Amer. Math. Soc. 120 (1996). MR 97j:16009

[12] D. Happel and D. Vossieck, Minimal algebras of infinite type with preprojective component, Manuscr. Math. 42 (1983), 221-243. MR 84m:16022

[13] O. Kerner, Tilting wild algebras, J. London Math. Soc. 39 (1989), 29-47. MR 90d:16025

[14] O. Kerner and A. Skowroński, On module categories with nilpotent infinite radical, Compositio Math. 77 (1991), 313-333. MR 92b:16011

[15] H. Lenzing and A. Skowroński, Quasi-tilted algebras of canonical type, Colloq. Math. 71 (1996), 161-181. MR 97j:16019

[16] S. Liu, Tilted algebras and generalized standard Auslander-Reiten components, Archiv. Math. 61 (1993), 12-19. MR 94g:16017

[17] S. Liu, Semi-stable components of an Auslander-Reiten quiver, J. London Math. Soc. 47 (1993), 405-416. MR 94a:16024

[18] S. Liu, On short cycles in a module category, J. London Math. Soc. 51 (1995), 62-74. MR 96e: 16015

[19] J. A. de la Peña and B. Tomé, Iterated tubular algebras, J. Pure Appl. Algebra 64 (1990), 303-314. MR 91h:16028

[20] I. Reiten, A. Skowroński and S. O. Smalø, Short chains and short cycles of modules, Proc. Amer. Math. Soc. 117 (1993), 343-354. MR 93d:16018

[21] C. M. Ringel, Tame algebras, in: Representation Theory I, Lecture Notes in Math. 831 (Springer-Verlag, 1980), 137-287. MR 82j:16056

[22] C. M. Ringel, Tame algebras and integral quadratic forms, Lecture Notes in Math. 1099 (Springer-Verlag, 1984). MR 87f:16027

[23] A. Skowroński, Selfinjective algebras of polynomial growth, Math. Ann. 285 (1989), 177-199. MR 90k: 16024

[24] A. Skowroński, Algebras of polynomial growth, in: Topics in Algebra, Banach Center Publications, Vol. 26, Part 1 (PWN, Warszawa, 1990), 535-568. MR 93k:16026

[25] A. Skowroński, Generalized standard Auslander-Reiten components, J. Math. Soc. Japan 46 (1994), 517-543. MR 95d:16022

[26] A. Skowroński, On the composition factors of periodic modules, J. London Math. Soc. 49 (1994), 477-492. MR 95g:16011

[27] A. Skowroński, Minimal representation-infinite artin algebras, Math. Proc. Camb. Phil. Soc. 116 (1994), 229-243. MR 95h:16015

[28] A. Skowroński, Cycle-finite algebras, J. Pure Appl. Algebra 103 (1995), 105-116. MR 96i: 16023

[29] A. Skowroński, Module categories over tame algebras, in: Representation Theory of Algebras and Related Topics, CMS Conference Proc. 19 (AMS, 1996), 281-313. MR 97e:16033

[30] A. Skowroński, Simply connected algebras of polynomial growth, Compositio Math. 109 (1977), 99-133. CMP 98:02

[31] A. Skowroński, Tame quasi-tilted algebras, J. Algebra 203 (1998), 470-490. CMP 98:12

Faculty of Mathematics and Informatics, Nicholas Copernicus University, Chopina 12/18, 87-100 TORú́, Poland

E-mail address: skowron@mat.uni.torun.pl 